\title{
Luminescent solar concentrators - A low cost photovoltaics alternative
}

\author{
Wilfried G.J.H.M. van Sark \\ Science, Technology and Society, Copernicus Institute, Utrecht University, Utrecht, The Netherlands
}

\section{A R T I C L E I N F O}

Article history:

Available online 3 February 2012

\section{Keywords:}

Luminescent solar concentrator

Organic dye

Quantum dot

Low-cost photovoltaics

Spectrum conversion

\begin{abstract}
A B S T R A C T
The development and current status of luminescent solar concentrators is reviewed. These solar concentrators generally consist of transparent polymer sheets doped with luminescent species; presently mainly organic dye molecules are used as luminescent species, however semiconductor nanocrystals are gaining interest. Direct and diffuse incident sunlight is absorbed by the luminescent species and emitted at red- shifted wavelengths with high quantum efficiency. Optimum design ensures that a large fraction of emitted light is trapped in the sheet, which travels to the edges where it can be collected by one or more mono- or bifacial solar cells, with minimum losses due to absorption in the sheet and re-absorption by the luminescent species. Optimized luminescent solar concentrators are predicted to offer potentially lower cost per unit of power compared to conventional solar cells. Various design and material aspects will be discussed using thermodynamic and ray-trace modeling techniques and recent experimental results are presented.
\end{abstract}

(c) 2012 Elsevier Ltd. All rights reserved.

\section{Introduction}

The key driver in global solar photovoltaic energy technology today still is lowering the cost per unit of power generated, in which fundamental research toward reaching high conversion efficiencies goes hand in hand with research in lowering production cost. Enormous progress has been made in the past decades, leading to present module cost between 1 and 2 Euro/Wp, depending on technology. This will bring electricity cost close to the price of $\sim 0.2$ Euro/kWh consumers pay today, but is still far off from bulk electricity generation cost of $0.02-0.04$ Euro/kW for conventional, fossil-fuel based power plants.

Conversion of the incident solar spectrum to monochromatic light would greatly increase the efficiency of solar cells. Down shifting was suggested in the late 1970s to be used in so-called luminescent solar concentrators (LSC) [1,2]. To these LSCs one or more solar cell(s) were attached. LSCs consist of a highly transparent plastic, in which luminescent species, usually organic dye molecules, are dispersed, see Fig. 1. These dyes absorb incident light and emit it at a red-shifted wavelength, with high quantum efficiency. Internal reflection ensures collection of part of the emitted light in the solar cell(s) at the side(s) of the plastic body. The energy of the emitted photons ideally is only somewhat larger than the band gap of the attached solar cells, to ensure near-unity conversion efficiency, and an increase in solar cell efficiency would result.

E-mail address: w.g.j.h.m.vansark@uu.nl.
Also, it was suggested to use a number of different organic dye molecules of which the re-emitted light was matched for optimal conversion by different solar cells. This is similar to using a stack of multiple solar cells, each sensitive to a different part of the solar spectrum. Efficiencies of $\sim 30 \%$ were expected [3].

The development of LSCs began as an alternative approach to lower the costs of PV. Unlike standard solar concentrators both direct and diffuse light is concentrated, without the need for expensive tracking, by a factor of typically 5-10, and smaller silicon (or other more expensive) solar cells can be used. As the cost of the transparent plastic is expected to be much lower than the area cost of the solar cell the cost per Watt-peak (Wp) of an LSC is lower compared to the cost of a planar silicon solar cell. Also, LSCs are of special interest for building integrated PV applications.

Research over the past three decades has been focused on [5]: 1) absorbing all photons with wavelength $>950 \mathrm{~nm}$, and emit them red-shifted at $\sim 1000 \mathrm{~nm}$, for use with c-Si solar cells; 2 ) have a low spectral overlap between absorption and emission spectra to minimize re-absorption losses; 3) have near-unity luminescence quantum yield; 4) have low escape cone losses; 5) are stable outdoors for longer than 10 years; 6 ) are easy to manufacture at low cost. Much progress has occurred, which is illustrated by the recent efficiency record of 7.1\% [6] for organic dyes in PMMA, as a result of modelling optimization study [4]. In addition, nanocrystals or quantum dots are increasingly used replacing the organic dyes; LSCs are then referred to as Quantum Dot Concentrators (QDCs) [7].

In this contribution we will present recent modelling and experimental results illustrating requirements for design and 

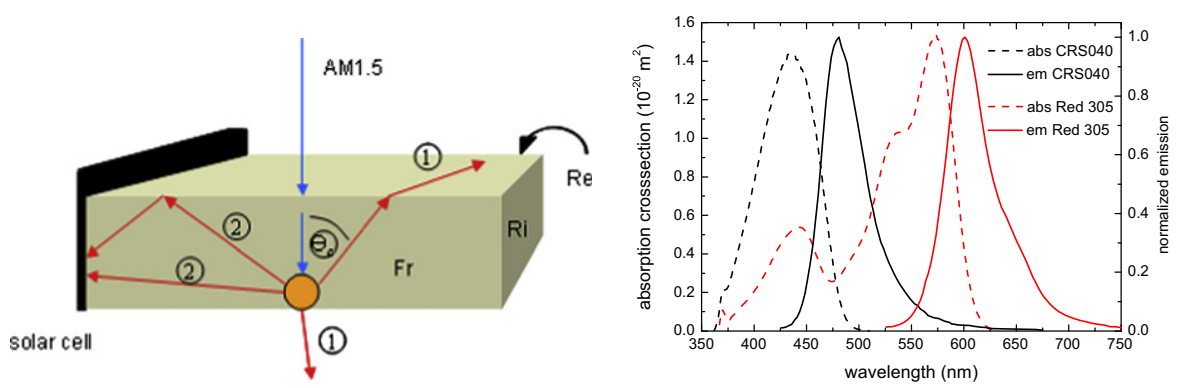

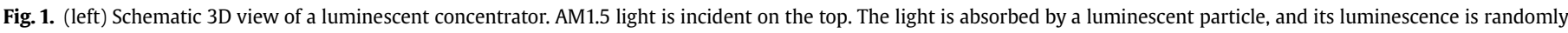

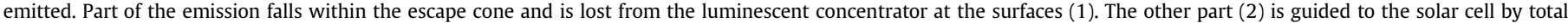
internal reflection [4]. (right) Absorption cross-section and normalized emission spectra of two fluorescent dyes, illustrating the Stokes' shift.

materials to ensure optimal performance, while at the same time keeping the cost low.

\section{Basic principle}

The principle of operation of the LSC is illustrated in Fig. 1. Light is incident on the top of a plastic plate with refractive index $n$. In this plate luminescent species are present, that absorb the light and isotropically emit it at a wavelength, which is generally red-shifted as a result of the Stokes' shift of the particular luminescent specie (see also Fig. 1). The Stokes' shift is defined as the difference between maxima in absorption and emission spectra, related to the same transition. Part of the emission falls within the escape cone (determined by the critical angle $\left(\theta_{c}\right)$ ) and is lost from the luminescent concentrator at the surfaces (1). The critical angle is defined by

$\theta_{c}=\sin ^{-1}\left(\frac{1}{n}\right)$

The other part (2) of the luminescence is guided to the solar cell by total internal reflection, which is lossless within the angular range. The other sides of the plastic plate should be covered with mirrors to trap photons outside the angular range. The efficiency of the complete LSC including solar cell $\eta_{L S C}$ can be expressed as the product of the optical efficiency $\eta_{\text {opt }}$ of the LSC plate and the efficiency of the solar cell $\eta_{P V} ; \eta_{o p t}$ is written as [8]:

$\eta_{\text {opt }}=(1-R) \eta_{a b s} \eta_{L Q E} \eta_{S} \eta_{\text {trap }} \eta_{\text {mat }}\left(1-\eta_{\text {self }}\right) \eta_{T I R}$

with $R$ the surface reflection coefficient, $\eta_{a b s}$ the absorption efficiency (the fraction of sunlight absorbed by the luminescent species), $\eta_{L Q E}$ the quantum efficiency of the luminescent species, $\eta_{S}$ the Stokes' efficiency, which is the energy loss between absorption and emission, $\eta_{\text {trap }}$ the efficiency of trapping of light (fraction of light outside the escape cone), $\eta_{\text {mat }}$ the efficiency of transmission of light in the plastic matrix, $\eta_{\text {self }}$ the efficiency of self-absorption of the luminescent species, $\eta_{T I R}$ the efficiency of total internal reflection.

The loss due to surface reflection equals $[(n-1) /(n+1)]^{2}$, the Fresnel reflection loss. For a polymethylmethacrylate (PMMA) plastic with $n=1.49$ this loss amounts to 0.0387 , and applying an anti-reflection coating can lower it. The fraction of sunlight that can be absorbed by the luminescent species depends on the specific material; organic dyes have narrow absorption bands, in contrast to QDs. An example is given in Fig. 1, in which the absorption and emission spectra are depicted for Fluorescence Yellow CRSO40 (coumarine dye, from Radiant Color) and Lumogen F Red305 (perylene, from BASF). The loss due to non-unity quantum efficiency is typically low for organic dyes, while QDs may have quantum efficiency values between 20 and $80 \%$. The Stokes' shift between absorption and emission is required to be small to minimize energy loss; on the other hand it should be large to minimize the overlap between the emission and absorption spectrum causing re-absorption (or self-absorption). The light trapping efficiency can be written as $(1 / n) \sqrt{ }\left(n^{2}-1\right)$, which equals 0.741 for PMMA; the critical angle is $\theta_{c}=42.2^{\circ}$. This efficiency can be enhanced by the use of selective mirrors or mirrors based on photonic. The transmission efficiency in the matrix depends on scattering or absorption in the matrix; an absorption coefficient of $1 \mathrm{~m}^{-1}$ is typical. Self-absorption in general leads to a red-shift of the emission spectrum, as well as intensity loss. Total internal reflection loss depends on surface quality. Taking into account all these losses, typical LSC efficiencies range from 3 to $8 \%$.

The maximum efficiency of the LSC was analyzed using the Shockley-Queisser principle of detailed balance. Efficiency values as high as 33\% were calculated, due to the presence of a perfect band pass mirror at the top surface [9]. The maximum concentration possible has been determined to be dependent on Stokes' shift of the luminescent species. For the Lumogen F Red 300 dye from BASF it can be calculated that $C=119$, as absorption and emission maxima are 578 and $613 \mathrm{~nm}$, respectively [3]. This maximum is one order of magnitude larger than typically used in practical LSCs.

\section{Modelling}

\subsection{Existing models}

Several groups have reported on the modelling of the LSC. Principally two different approaches are used, a thermodynamic detailed balance model which is based on the radiative energy transfer between mesh points in the concentrator plate [7], and a ray-tracing model in which every incoming photon is tracked and its fate determined [10]. Both methods yield similar results, and are also in excellent agreement with experiments on test devices. With the thermodynamic model, requiring only a small amount of input parameters, results can be obtained quickly; however geometries are limited to block shapes only. In addition, only single absorbing species can be modelled presently. Ray-trace models are computationally expensive, as a large number of luminescent species have to be considered, as well as a large number of rays to obtain accurate results; however, simpler procedures involving absorption coefficients allow for much faster modelling. Due to the present versatility of the ray-trace model [10], a parametric analysis of an LSC is performed using this model.

\subsection{Parametric analysis}

Ray-tracing has been used to perform a parametric analysis for a $5 \times 5 \mathrm{~cm}^{2}$ planar LSC (with a solar cell on one side, and mirrors 
at the remaining side and bottom surfaces) to find attainable LSC efficiencies for various mirror configurations, plastic matrix material properties, type of solar cell and dye. The concentrator consists of a PMMA plate (refractive index $n=1.49$, absorption $1.5 \mathrm{~m}^{-1}$ ) doped with two luminescent dyes, CRS040 from Radiant Color and Lumogen F Red 305 from BASF (Fig. 1), with an LQE of 95\%. The conversion efficiency of this LSC was modelled to be $2.45 \%[4]$.

Direct mirrors or a mirror with an air-gap between the mirror and the LSC were modelled, as well as different reflectivity values and specular or Lambertian mirror types. Without a side mirror, rays within the escape cone leave the LSC at that side; rays outside the escape cone are subject to total internal reflection, see Fig. 2(a). When a direct mirror is applied, the total internal reflection disappears, and all rays reflect with the reflection coefficient of the mirror, see Fig. 2(b). For the rays outside the escape cone this leads to a reduction in the reflection, and thus lower power conversion efficiency. The use of an "air-gap mirror", i.e., an air-gap between mirror and LSC, combines total internal reflection with reflection of the escaping rays, see Fig. 2(c). The results of the calculations show a maximum efficiency of $2.94 \%$ for the case with $97 \%$ reflective airgap mirrors (e.g. 3M adhesive visible mirror foil, with a reflection of 97\%) at the sides and a 97\% reflective Lambertian air-gap mirror (e.g. standard integrating sphere material with a reflection of 97\%) at the bottom.

In a similar manner the background absorption of the polymer was varied in the absorption and emission range of the dye from the current value of $1.5 \mathrm{~m}^{-1}$ to a value of $10^{-3} \mathrm{~m}^{-1}$. We found that the efficiency of the LSC can be increased to slightly over $3.4 \%$. An additional increase of the refractive index of the polymer from 1.49 to 1.7 would result in an increase in $\eta$ to almost $3.8 \%$, as a result of the fact that a higher refractive index leads to a larger fraction of the emitted dye luminescence being trapped in the concentrator plate. Note, however, that a polymer with the combination of a refractive index of 1.7 and an optical absorption of $10^{-3} \mathrm{~m}^{-1}$ is not currently available.

One major reason for the lower efficiency compared to early work is that the used solar cells are not optimized for the emissionspectrum of the dye: mc-Si solar cells absorb all the light up to about $1100 \mathrm{~nm}$, but the dyes used in these LSCs emit around $650 \mathrm{~nm}$. It would therefore be beneficial to use a solar cell with a larger band gap, because these deliver the same current as the mc-Si cell, but at larger open circuit voltage $\left(V_{o c}\right)$. In Table 1 estimates are listed for attainable efficiencies $\eta$ for similar concentrator plates with different solar cells attached. Replacing the mc-Si cell with a GaAs or InGaP cell, will increase $\eta$ from $3.8 \%$ to 6.5 and $9.1 \%$, respectively (based on $V_{o c}$ (fillfactor) values of $0.58 \mathrm{~V}(0.83), 1.00 \mathrm{~V}$ (0.83), and $1.38 \mathrm{~V}(0.84)$, for mc-Si, GaAs, InGaP, respectively). Thus, the use of GaAs or InGaP cells will result in higher efficiencies, but these cells are more expensive.
Table 1

Efficiencies (in \%) for an LSC for various optimized parameters and solar cells.

\begin{tabular}{llll}
\hline Parameters & mc-Si & GaAs & InGaP \\
\hline $\begin{array}{l}\text { Fixed mirrors, 85\% reflectivity, dyes } \\
\quad \text { with 95\% LQE }\end{array}$ & 2.4 & 4.2 & 5.9 \\
$\begin{array}{l}97 \% \text { Reflectivity "air-gap mirrors" on } \\
\text { sides, and 97\% reflectivity }\end{array}$ & 2.9 & 5.1 & 7.1 \\
$\quad \begin{array}{l}\text { Lambertian mirror at bottom } \\
\text { Reduce background absorption of } \\
\quad \text { matrix from } 1.5 \mathrm{~m}^{-1} \text { to } 10^{-3} \mathrm{~m}^{-1}\end{array}$ & 3.4 & 5.9 & 8.3 \\
$\begin{array}{l}\text { Increase of refractive index from } \\
1.49 \text { to } 1.7\end{array}$ & 3.8 & 6.5 & 9.1 \\
\hline
\end{tabular}

\section{Experimental results}

Most of the early work has been reviewed [8]. The 4\% efficiency reached in the early period still is an important target to cross by present experimental efforts, especially for the larger $\left(40 \times 40 \mathrm{~cm}^{2}\right)$ sized plates. Another reason for low efficiencies was the lack of dyes emitting in the NIR; experimental data on quantum efficiency in the early period showed that the longer the emission wavelength, the lower the luminescence quantum efficiency. Stability of the LSC system is a prerequisite for long-term outdoor operation; LSC systems have been tested and were shown to be stable for a period of 2 years.

Recent efforts have lead to several groups surpassing the $4 \%$ limit, albeit for smaller area size. For example, Goldschmidt et al. [11] showed for a stack of two plates with different dyes, to which four GaInP solar cells were placed at the sides, that the conversion efficiency is $6.7 \%$; the plate was small $\left(4 \mathrm{~cm}^{2}\right)$, and the concentration ratio was only 0.8 . According to them the conversion efficiency was limited by the spectral range of the dyes used, and $13.5 \%$ efficiency could be reached if for the 650-1050 nm range the same quantum efficiency as for the $450-600 \mathrm{~nm}$ range could be realized. They also discuss the benefits of a photonic structure ('rugate filter') on top of the plate, to reduce the escape cone loss [11]. The use of these filters would increase the efficiency by $\sim 20 \%$, as was determined for an LSC consisting of one plate and dye. Currie et al. [12] projected by modelling conversion efficiencies as high as $6.8 \%$, for a tandem LSC based on two single LSCs that consist of thin layer of deposited organic dye molecules onto a glass plate to which a GaAs cell was attached. Using $\mathrm{CdTe}$ or $\mathrm{Cu}(\mathrm{In}, \mathrm{Ga}) \mathrm{Se}_{2}$ solar cells conversion efficiency projections of $11.9 \%$ and $14.5 \%$, respectively, were calculated. Slooff et al. [6] presented results on $50 \times 50 \times 5 \mathrm{~mm}^{3}$ PMMA plates in which both CRS040 and Red305 dyes were dispersed at 0.003 and $0.01 \mathrm{wt} \%$, respectively. The plates were attached to either mc-Si, GaAs or InGaP cells, and a diffuse reflector (97\% reflection) was used at the rear side of the plate. The highest efficiency measured was $7.1 \%$ for $4 \mathrm{GaAs}$ cells connected in parallel $7 \%$ if connected in series), which so far is the highest efficiency reported.

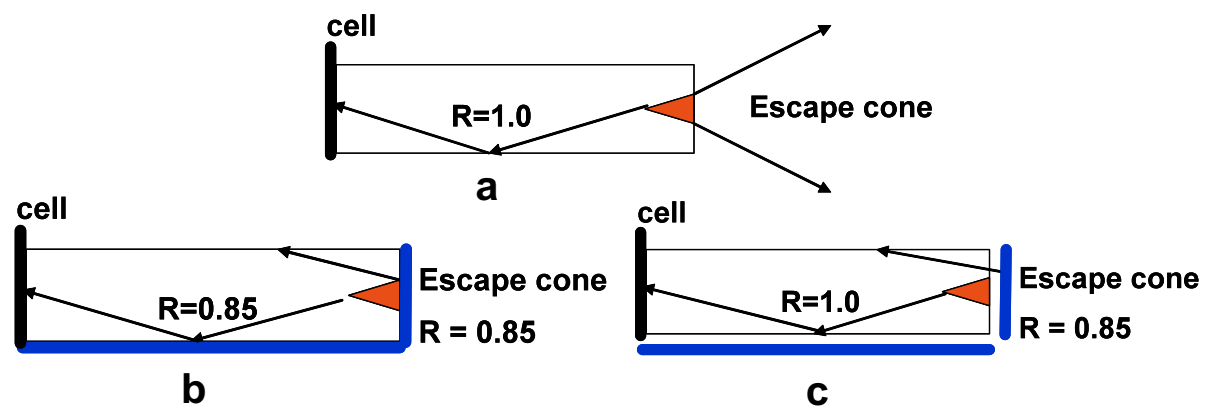

Fig. 2. Different mirror configurations that are used in the ray-tracing simulation. (a) no mirrors, (b) direct mirrors, and (c) air-gap mirrors [4]. 


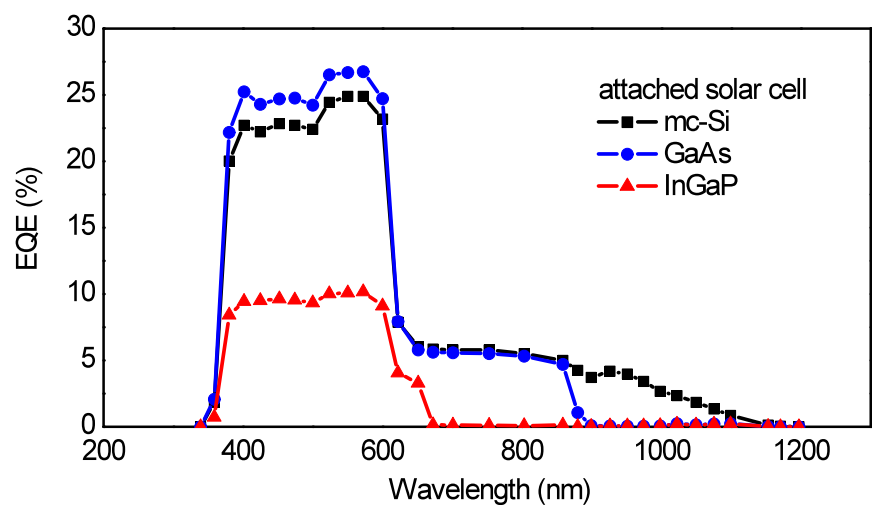

Fig. 3. External quantum efficiency of an LSC with mc-Si, GaAs or InGaP cell attached [6].

The external quantum efficiency of the LSC is shown in Fig. 3. The response between 400 and $600 \mathrm{~nm}$ clearly results from dye absorption. The response at higher wavelengths is due to scattering of incident light at the backside mirror. Similar plates were attached to a-Si:H solar cells, but the attained efficiency was $\sim 1 \%$ for two cells connected in parallel [13].

Quilitz et al. [14] recently addressed several problems regarding incorporation of QDs in an organic polymer matrix, viz. phase separation, agglomeration of particles leading to turbid plates, and luminescence quenching due to exciton energy transfer. QDCs were synthesized using CdSe core/multishell QDs (QE $=60 \%)$ and were dispersed in laurylmethacrylate (LMA), that subsequently was UVpolymerized. Transparent PLMA plates with QDs were obtained without any sign of agglomeration. To one side of this plate, a $5 \times 0.5 \mathrm{~cm}^{2} \mathrm{mc}$-Si solar cell was placed, and aluminium mirrors to all other sides. The short circuit current of the best QDC was $77 \mathrm{~mA} /$ $\mathrm{cm}^{2}$ under $1000 \mathrm{~W}$ illumination, nearly double to value of $40 \mathrm{~mA} /$ $\mathrm{cm}^{2}$ of a bare cell. The QDC efficiency was 3.5\%.

\section{Cost}

Widespread deployment of LSCs will only be possible if the cost per Watt-peak generated is lower than $1 € / \mathrm{Wp}$. LSC cost is composed of plate cost and solar cell cost, which are related to their respective areas, and thus are detailed in $€ / \mathrm{m}^{2}$. Currie et al. [12] use cost in $\$ / \mathrm{Wp}$, and they find that using a 50\$/Wp GaInP solar cell the geometrical gain should be as high as 50 . Hence, both solar cell cost should come down as well as geometrical gain increased. Using c-Si solar cells or proper band gap amorphous silicon cells should be preferred, as geometrical gains range from 2 to 10 . Bende et al. [15] have presented a cost-optimization study focusing on relative area cost and relative cost per unit of power, since they compare LSC with conventional PV cost. Assuming a realistic ratio between polymer-plate cost and PV area cost of 1 / 15 , they found that the lowest relative cost per unit of power can be as low as 0.35 to 0.55 for a combined plate thickness and length of $1 \mathrm{~mm}$ and $0.25 \mathrm{~m}$, and $10 \mathrm{~mm}$ and $0.55 \mathrm{~m}$, respectively. Thus, LSC cost could be $\sim 1 / 3$ of c-Si cost, in terms of relative cost per unit of power. As these optimized costs depend critically on the ratio between polymer-plate cost and PV area cost, a sensitivity study showed that this ratio should at least be lower than $1 / 5$ for LSCs to be economically viable.

\section{Conclusion}

In this contribution a short overview of modelling and experimental work performed on LSCs was presented. The renewed interest in the LSCs, since the pioneering work in the 1970s and 1980s, has lead to the development of thermodynamic and raytrace models, which equally well describe the performance of LSCs. Also, new dyes, and more importantly, new quantum dots have been developed and have been included in the polymer concentrators. Stability tests for over 2 years have shown that good candidates exist for use in commercial type LSCs. Future research should be directed towards lowering escape cone losses, lowering the absorption in the matrix, as well as re-absorption. Further, the absorption range should be enlarged in order to realize efficiencies well above $10 \%$; this may be possible using appropriately tuned quantum dots in a single plate configuration, or multi plate hybrid stacks in which both organic and inorganic materials are combined.

\section{References}

[1] Goetzberger A, Greubel W. Solar energy conversion with fluorescent collectors. Applied Physics 1977;14:123-39.

[2] Weber WH, Lambe J. Luminescent greenhouse collector for solar radiation. Applied Optics 1976;15:2299-300.

[3] Smestad G, Ries H, Winston R, Yablonovitch E. The thermodynamic limits of light concentrators. Solar Energy Materials 1990;21:99-111.

[4] Van Sark WGJHM, Barnham KWJ, Slooff LH, Chatten AJ, Büchtemann A, Meyer A, et al. Luminescent solar concentrators - a review of recent results. Optics Express 2008;16:21773-92.

[5] Rowan BC, Wilson LR, Richards BS. Advanced material concepts for luminescent solar concentrators. IEEE Journal of Selected Topics in Quantum Electronics 2008; 14:1312-22.

[6] Slooff LH, Bende EE, Burgers AR, Budel T, Pravettoni M, Kenny RP, et al. A luminescent solar concentrator with $7.1 \%$ power efficiency. Physica Status Solidi (RRL) 2008;2:257-9.

[7] Chatten AJ, Barnham KWJ, Buxton BF, Ekins-Daukes NJ, Malik MA. Quantum dot solar concentrators. Semiconductors 2004;38:609-17.

[8] Petrova-Koch V, Goetzberger A, editors. In high-efficient low-cost photovoltaics - recent developments. Heidelberg, Germany: Springer; 2008.

[9] Rau U, Einsele F, Glaeser GC. Efficiency limits of photovoltaic fluorescent collectors. Applied Physics Letters 2003;87:171101.

[10] Burgers AR, Slooff LH, Kinderman R, van Roosmalen JAM. Modelling of luminescent concentrators by ray-tracing. In: Proceedings Twentieth European photovoltaic solar energy Conference, Barcelona, Spain, 6-10 June 2005; 2005. p. 394-7.

[11] Goldschmidt JC, Peters M, Boüsch A, Helmers H, Dimroth F, Glunz SW, et al. Increasing efficiency of fluorescent concentrator systems. Solar Energy Materials Solar Cells 2009;93:176-82.

[12] Currie MJ, Mapel JK, Heidel TD, Goffri S, Baldo MA. High-efficiency organic solar concentrators for photovoltaics. Science 2008;321:226-8.

[13] Farrell DJ, van Sark WGJHM, Velthuijsen ST, Schropp REI. Using amorphous silicon solar cells to boost the viability of luminescent solar concentrators. Physica Status Solidi C 2009;7:1045-8.

[14] Bomm J, Büchtemann A, Chatten AJ, Bose R, Farrell DJ, Chan NLA, et al. Fabrication and full Characterisation of state-of-the-artCdSe quantum dot luminescent solar concentrators. Solar Energy Materials and Solar Cells 2011; 95:2087-94.

[15] Bende EE, Burgers AR, Slooff LH, Van Sark WGJHM, Kennedy M. Cost and efficiency optimisation of the fluorescent solar concentrator. In: Proceedings Twenty third European photovoltaic solar energy Conference, Valencia, Spain, 1-5 September 2008; 2008. p. 461-9. 